\title{
Comparison of the Kansas City Cardiomyopathy Questionnaire and Minnesota Living With Heart Failure Questionnaire in Predicting Heart Failure Outcomes
}

\author{
Derek Yee, MD, Eric Novak, MS, Anne Platts, MD, Michael E. Nassif, MD, Shane J. LaRue, \\ MD, Justin M. Vader, MD \\ Washington University School of Medicine, St. Louis, Missouri
}

\begin{abstract}
Patient reported outcome measures (PROMs) are relevant independent outcomes in heart failure (HF) care and are predictive of subsequent hospitalization and death in HF. The Kansas City Cardiomyopathy Questionnaire (KCCQ) and the Minnesota Living with Heart Failure Questionnaire (MLHFQ) are the 2 most widely adopted PROMs specific to HF. We compared their prognostic abilities in a prospective cohort of HF patients. A prospective cohort of subjects from a single-center registry was analyzed with regard to baseline KCCQ and MLHFQ scores and the outcomes of death, transplant, or left ventricular assist device implantation and hospitalization. A total of 516 subjects with reduced left ventricular ejection fraction (HFrEF) and 151 subjects with preserved left ventricular ejection fraction (HFpEF) were included. Discrimination was assessed using c-statistics based on time-to-event analyses and receiver-operator curves. The additive contribution of MLHFQ was assessed through the change in c-statistic, incremental discrimination index, and category free net reclassification index. Overall, KCCQ was superior to MLHFQ for predicting death/transplant/VAD (c-statistic 0.702 (0.666-0.738) and 0.658 (0.621-0.695) respectively, p-value for difference $<0.001$ ) and hospitalization (c-statistic 0.640 (0.613-0.666) and 0.624 (0.597-0.651), respectively, p-value for difference 0.022). However, this difference was statistically non-significant in the HFpEF group alone. When analyzing the additional prognostic information afforded by adding MLHFQ to KCCQ in the overall, HFrEF, and HFpEF groups there was no significant improvement, although adding KCCQ to MLHFQ did significantly improve risk stratification. Scoring based upon the abbreviated KCCQ-12 did not reduce the prognostic accuracy of KCCQ. In conclusion, KCCQ is more prognostic of death/ transplant/LVAD and hospitalization than MLHFQ in a combined cohort of patients with HFrEF and HFpEF, although the effect in HFpEF was less pronounced. KCCQ should be the preferred PROM for patients with HF if prognostication is a desired goal of using the PROMs.
\end{abstract}

\footnotetext{
Correspondence: Justin M. Vader MD, Cardiovascular Division, Washington University in St. Louis. 660 South Euclid Ave, Campus Box 8086 St. Louis, MO 63110. Fax: 314-362-4619. Phone: 314-362-1291., jvader@wustl.edu.

Disclosures: No relevant disclosures.

Publisher's Disclaimer: This is a PDF file of an unedited manuscript that has been accepted for publication. As a service to our customers we are providing this early version of the manuscript. The manuscript will undergo copyediting, typesetting, and review of the resulting proof before it is published in its final citable form. Please note that during the production process errors may be discovered which could affect the content, and all legal disclaimers that apply to the journal pertain.
} 


\section{Keywords}

Heart Failure; Patient Reported Outcome Measure

The Kansas City Cardiomyopathy Questionnaire (KCCQ) and the Minnesota Living with Heart Failure Questionnaire (MLHFQ) are two of the most widely used and validated questionnaires for assessing heart failure specific quality of life ${ }^{1-5}$. These questionnaires, now validated in HF patients with both reduced and preserved ejection fraction (HFrEF, HFpEF), have primarily been used in clinical research and less commonly in clinical practice due to their time-intensive nature and lack of specific reimbursement ${ }^{6-9}$. A recent review of patient reported outcome measures (PROM) in HF concluded that KCCQ and MLHFQ are the best suited outcome measures for prospective study in the delivery of patient care ${ }^{2}$. In addition to characterizing patient symptom burden, it is compelling that these scores might also predict major clinical outcomes such as hospitalization, progression of heart failure, and death. Understanding the prognostic value of these scores might improve their usefulness in clinical practice, where treatment options are often tailored to prognosis. To date, there has been little direct comparison of KCCQ and MLHFQ in realworld populations of HF patients, and in fact, some trials have used both PROMs, increasing time and financial costs in the absence of a clear additive benefit ${ }^{1,2,10-13}$. We sought to compare the predictive ability of KCCQ and MLHFQ in a prospective cohort study of realworld HFrEF and HFpEF patients.

\section{METHODS}

Patient data were obtained from the Washington University Heart Failure Registry, a large, prospective registry of inpatients and outpatients that have a clinical diagnosis of heart failure evaluated at Washington University School of Medicine or Barnes-Jewish Hospital, St. Louis, MO. Detailed patient characteristics were collated including demographics, vital signs, onset of diagnosis of heart failure, New York Heart Association (NYHA) classification status, comorbidities, health status, and hospitalizations. Each patient signed informed consent to participate. The study was approved by the Washington University Institutional Review Board.

Patients were assessed for clinical events and health status at the time of enrollment and followed to a maximum of two years. Disease-specific health status was quantified with KCCQ, KCCQ-12, and MLHFQ. The KCCQ is a 23-item self-administered questionnaire that quantifies multiple domains of patients' HF-related health status including physical limitation, symptom stability, symptom frequency, symptom burden, self-efficacy, quality of life, and social limitations ${ }^{3}$. Items are scored from 0 to 100 with 0 representing the worst and 100 the best possible functional status. The KCCQ-12 is a recently developed, shortened form of the KCCQ that contains 12 items compared to the 23 items of the original instrument. It has been shown to preserve the validity, reliability, and prognostic ability of the original instrument ${ }^{14}$. MLHFQ is a 21 -item self-administered questionnaire with the overall score based upon physical and emotional dimensions of how heart failure affects patients' lives ${ }^{4}$. Items are individually scored from zero to five, with a higher score 
representing more significant impairment in that particular aspect of disease-specific quality of life. KCCQ and MLHFQ responses were filled out by the study subjects and tabulated by the study coordinators.

Survival and events were tracked from enrollment into the heart failure registry until event occurrence or last known follow-up. The principal outcome of interest is the composite of death, transplant, or ventricular assist device (VAD) implant. The secondary outcome of interest was hospitalization. Patients were followed for a maximum of 2 years. Clinical outcomes after enrollment were obtained either by in-person clinic interviews or by telephone calls at home. Additionally, chart reviews using the Washington University School of Medicine / Barnes-Jewish Hospital electronic medical records were performed at 6, 12, 18, and 24 months. Patients were grouped into HFrEF (EF $\leq 40 \%$ ) and HFpEF (EF $250 \%$ ) for principal and secondary analysis. We employ a similar reasoning for choosing these values as the cutoffs for HFrEF and HFpEF as Joseph et al. ${ }^{6}$. Descriptive statistics were used to describe patient characteristics. Summary statistics for continuous and categorical variables were generated as appropriate. All statistical analysis was conducted in SAS v.9.4 (SAS Institute Inc., Cary, NC) and R using the packages survC1, survivalROC, and Hmisc.

KCCQ scores and MLHFQ scores were divided into quartiles. Comparisons between KCCQ quartiles and MLHFQ quartiles were done using analysis of variance (ANOVA) for continuous variables and the chi-square test for categorical data. All ordinal and non-normal data were compared using the Kruskal-Wallis test. A multivariable Cox proportional hazards model was used to examine the association between the primary and composite outcome and KCCQ and MLHFQ. The following adjustment variables were selected a priori: age, gender, race, body mass index (BMI), heart rate, systolic blood pressure (SBP), estimated glomerular filtration rate (eGFR), serum sodium concentration (Na), left ventricular ejection fraction (LVEF), and a history of myocardial infarction, atrial fibrillation, percutaneous coronary intervention (PCI), diabetes mellitus, hypertension, hyperlipidemia, stroke or transient ischemic attack, and smoking status. For each measure, the risk of primary outcome was described by the hazard ratio and corresponding 95\% confidence interval resulting from the appropriate Cox model. Missing data were imputed and multiple (5) data sets created, each using a sequential imputation algorithm. The discriminant function method was used to impute categorical variables. The regression predictive mean matching method was used to impute continuous variables.

The ability of KCCQ and MLHFQ to discriminate events from non-events was determined using c-statistics based on time-to-event analyses ${ }^{15}$. Additionally, Receiver Operating Characteristic (ROC) curves were created for each PROM within HFpEF and HFrEF groups, separately. The improvement in predictive ability from adding MLHFQ to KCCQ was evaluated by the category-free Net Reclassification Improvement (NRI) and the Integrated Discrimination Improvement (IDI). The NRI measures the correctness of reclassification based on event probabilities, and the IDI measures the improvement in discrimination slope which evaluates improvement in average sensitivity ${ }^{16,17}$. Both NRI and IDI were evaluated at 2 years post-enrollment. 


\section{RESULTS}

A total of 738 patients with completed KCCQ and MLHFQ were available for analysis. Of these, 516 (69.9\%) had LVEF $\leq 40 \%$ (HFrEF) and 151 (20.5\%) had LVEF $250 \%$ (HFpEF). A smaller number of 71 subjects (9.6\%) with an LVEF between $40 \%$ and $50 \%$ (HF with intermediate LVEF) were not analyzed. Full baseline characteristics of the participants are included in the supplementary tables [Tables A1 and A2]. Participants were enrolled between March 2010 and September 2012, providing an average follow-up time of 16.6 \pm 6.7 months. There were minimal amounts of missing data with only $10.1 \%$ missing one or more baseline characteristics. The most frequently missing variable was eGFR, which was missing in $2 \%$ of the patients.

KCCQ and MLHFQ score quartiles were highly correlated with many pre-specified variables, most notably with NYHA class ( $\mathrm{p}<0.001$ for both) (Tables 1 and 2). KCCQ scores in the first quartile corresponded to the majority of patients in NYHA Class III and IV (79\%) whereas a KCCQ score in the fourth quartile corresponded to the majority of patients in NYHA Class I and II (94\%). Conversely, a MLHFQ score in the first quartile corresponded to the majority of patients in NYHA Class I and II (89\%) whereas a MLHFQ score in the fourth quartile corresponded to the majority of patients in NYHA Class III and IV (72\%).

KCCQ and MLHFQ scores were strongly associated with the risk of death/transplant/VAD, with an unadjusted HR of 0.876 (per 5 point increase, p<0.001) for KCCQ and 1.099 (per 5 point increase, $\mathrm{p}<0.001$ ) for MLHFQ. When subdividing by EF, both KCCQ and MLHFQ were strongly associated with the risk of death/transplant/VAD in patients with EF 0-40 (per 5 point increase, HR 0.875 with $\mathrm{p}<0.001$, and HR 1.091 with $\mathrm{p}<0.001$, respectively) and EF 50-100 (per 5 point increase, HR 0.883 with $\mathrm{p}=0.005$, and HR 1.107 with $\mathrm{p}=0.015$, respectively) but not EF 41-49 (per 5 point increase, HR 0.936 with $\mathrm{p}=0.31$, and HR 1.068 with $\mathrm{p}=0.24$, respectively). In a multivariable Cox proportional hazards model incorporating the KCCQ score and key clinical predictors selected a priori, the KCCQ was significantly correlated with the composite outcome (HR=0.89/5-point increase in KCCQ score), as were heart rate, history of stroke/TIA, SBP, eGFR, and Na, all with $\mathrm{p}<0.001$ [Table 3]. Similarly, in a multivariable Cox proportional hazards model, MLHFQ scores were also highly correlated with the composite outcome (HR=1.08/5-point increase in MLHFQ score), as were heart rate, history of stroke/TIA, SBP, eGFR, and Na, all with $\mathrm{p}<$ 0.001 [Table 4]. Additionally, we analyzed the interaction of heart failure type (HFpEF, HFrEF, intermediate HF) with the impact of either KCCQ or MLHFQ on the combined outcome in Cox proportional hazards models, and there was no significant interaction. This analysis is described in the supplemental tables [Tables A3 and A4].

Outcome event frequencies were generated and categorized overall, by HFrEF, and HFpEF [Table 5]. For the outcome of death, transplant or VAD implant within 2 years, KCCQ was superior to MLHFQ in discrimination overall ( $c=0.702$ vs. $c=0.658, p<0.001$ ) and in HFrEF ( $c=0.696$ vs. $c=0.644, p<0.001$ ), but there was no statistical difference among HFpEF patients ( $\mathrm{c}=0.685$ vs. $\mathrm{c}=0.660, \mathrm{p}=0.67$ ) [Figures 1 and 2]. As with the long form KCCQ, the short form KCCQ-12 was superior to MLHFQ in discrimination overall ( $\mathrm{c}=0.704$ vs. $\mathrm{c}=0.658, \mathrm{p}<0.001)$ and in HFrEF ( $c=0.699$ vs. $\mathrm{c}=0.644, \mathrm{p}<0.001)$ but not $\operatorname{HFpEF}(\mathrm{c}=0.684$ 
vs. $\mathrm{c}=0.660, \mathrm{p}=0.58)$. In an analysis of hospitalization at 2 years, KCCQ was superior to MLHFQ in discrimination overall ( $c=0.640$ vs. $c=0.624, \mathrm{p}=0.022$ ); however, in an analysis of subgroups of preserved and reduced $\mathrm{EF}$, this difference did not attain nominal significance (HFrEF ( $c=0.636$ vs. $\mathrm{c}=0.620, \mathrm{p}=0.06$ ); $\mathrm{HFpEF}$ ( $\mathrm{c}=0.628$ vs. $\mathrm{c}=0.612, \mathrm{p}=0.35$ )). These patterns were maintained with KCCQ-12 (overall ( $c=0.644$ vs. $c=0.624$, $\mathrm{p}=0.004$ ); HFrEF ( $c=0.640$ vs. $\mathrm{c}=0.620, \mathrm{p}=0.026) ; \mathrm{HFpEF}$ ( $\mathrm{c}=0.639$ vs. $\mathrm{c}=0.612, \mathrm{p}=0.24)$ ).

In light of the overall superior prognostic performance of KCCQ, the incremental change in prognostic ability gained by adding MLHFQ to KCCQ was assessed. With regard to the endpoint of death/transplant/VAD, the combined c-statistic using MLHFQ added to KCCQ was calculated. In all cases there was minimal increase: overall ( $c=0.705$, increase in cstatistic $=0.003)$, in HFrEF ( $c=0.702$, increase in $\mathrm{c}$-statistic $=0.006)$, and HFpEF ( $c=0.688$, increase in c-statistic $=0.002$ ). Furthermore, there was no significant improvement in prognostic capability as measured by the category-free NRI (overall $-3.6 \%, \mathrm{p}=0.68$; HFrEF $4.4 \%, \mathrm{p}=0.65$; HFpEF $-20.7 \%, \mathrm{p}=0.43$ ) or IDI (overall 0.002, $\mathrm{p}=0.17$; HFrEF 0.004, $\mathrm{p}=0.10$; HFpEF 0.001, $\mathrm{p}=0.58$ ) when adding MLHFQ to KCCQ. For hospitalization, the combined c-statistic using MLHFQ added to KCCQ was unchanged overall ( $c=0.670$, increase in cstatistic $=0.001)$, in HFrEF ( $c=0.663$, increase in $c$-statistic $=0.000)$ and $\operatorname{HFpEF}(c=0.636$, increase in c-statistic $=0.000$ ). Similar to the analysis of death/transplant/VAD, there was no significant improvement by NRI (overall $6.6 \%, \mathrm{p}=0.35$; HFrEF $0.1 \%, \mathrm{p}=0.98$; HFpEF $-20.7 \%, \mathrm{p}=0.40$ ) or IDI (overall $<0.001, \mathrm{p}=0.35$; $\mathrm{HFrEF}<0.001, \mathrm{p}=0.73$; $\mathrm{HFpEF}<0.001$, $\mathrm{p}=0.70$ ) when adding MLHFQ to KCCQ.

\section{DISCUSSION}

With increasing incidence of HF and a shift towards reduction of HF hospitalization rates, more of the care of these patients will be on an outpatient basis ${ }^{18,19}$. Tools for the outpatient assessment of chronic HF are increasingly important, allowing clinicians to correlate patients' report of their health status to disease-specific health risk. PROs quantify the symptom burden in HF, but may also risk stratify patients' risks for death and hospitalization. This capacity can be valuable in clinical practice to both inform patients of their prognosis and to increase the intensity of therapy as prognosis worsens. Given that KCCQ and MLHFQ are both commonly used disease-specific PROs in clinical trials, understanding which better predicts prognosis can help define which may have more clinical utility in routine practice. In this study we found in a prospective cohort of patients with HF, both KCCQ and MLHFQ were independently associated with a combined outcome of death/VAD/transplant after adjustment for conventional risk factors. Comparing PROMs, we found that in both unadjusted and adjusted analyses that KCCQ was better able to predict death/LVAD/transplant and hospitalizations. It was also demonstrated that the short form KCCQ-12 score demonstrated similar prognostic performance as compared to the full KCCQ score.

PROMs among patients with HF at outpatient visits may be more sensitive to clinical status change than many widely adopted practices such as serial weights or brain natriuretic peptide (BNP) measurement ${ }^{20}$. Despite increasing evidence that PROMs could be relevant to the outpatient visit, concerns remain that these instruments are too time intensive ${ }^{21-23}$. 
On average, KCCQ takes between 5-8 minutes to complete, KCCQ-12 takes between 2-4 minutes, and MLHFQ takes between 5-10 minutes to complete ${ }^{3,4}$. It is important to know which PROM represents the best commitment of time.

While we found the predictive accuracy of KCCQ for the composite outcome of death/ transplant/VAD to be superior to MLHFQ overall and in the cohort of patients with HFrEF, this superiority was less evident in the cohort of HFpEF, although the smaller sample size may have limited our ability to confirm statistical significance. Given the overlap in many of the symptoms associated with HFpEF and HFrEF, the psychometric properties across both symptom questionnaires would be expected to be equally valid descriptors of patients' health status. Indeed, KCCQ was recently shown to have validity for stratifying risk of a combined outcome of death or hospitalization among HFpEF patients using the same registry as our study ${ }^{6}$. The present study may have been limited in its ability to test the differential prognostic capabilities of these PROMs in HFpEF for the outcome of death/ transplant/VAD due to a lower number of subjects and relatively lower event rate amongst patients with HFpEF compared to HFrEF. Additionally, the HFpEF population may have been more likely to experience non-HF causes for death or hospitalization, decoupling HF symptom inventories from the measured outcomes. Longer follow-up times and larger HFpEF study cohorts may be needed to observe a significant difference in the predictive abilities of KCCQ and MLHFQ for HF events in the HFpEF population. Ultimately, the results of this study may be used to inform clinical practice and the conduct of clinical research. When choosing a PROM for either routine patient care or clinical research, particularly in patients with HFrEF, KCCQ is superior to combined or preferential use of MLHFQ. Further, the short form KCCQ-12 appears to offer similar prognostic ability and less time to complete than the full KCCQ. Thus, in time-limited settings such as routine clinical practice, it would be reasonable to use KCCQ-12 as it is more convenient.

Our results should be interpreted in the context of certain limitations. First, KCCQ and MLHFQ were administered only at initial enrollment, preventing analysis of serial testing for test-retest reliability and time-varying trends. Second, this was a single-center study conducted within a large academic medical practice and was not designed to be a nationally representative sample. While the patient population is likely to be generalizable across other large academic centers, and baseline characteristics with this registry did show good representation by women and minorities, this sample inevitably represents some referral bias of patients expected to require consideration for advanced HF therapies. Finally, subjects included in the analysis were participants in a larger HF registry program and as willing volunteers, the quality of their responses to a PROM may be more thoughtful as compared to an unselected clinical population when using these PROMs as a matter of routine clinical practice. This said, the limitations should be expected to equally affect KCCQ and MLHFQ and would not be expected to bias the comparative accuracy of these PROMs.

In summary, this is the first study of which we are aware to directly compare the predictive validity of KCCQ and MLHFQ, the two most widely used PROMs in HF. We found that in a prospective cohort study of HF patients, both KCCQ and MLHFQ were predictive of a composite of death, transplant, or LVAD implant; as well as hospitalization. When comparing the two instruments, KCCQ consistently outperformed MLHFQ and addition of 
MLHFQ to KCCQ did not offer additional predictive ability for either death/transplant/VAD or hospitalization. Use of the abbreviated KCCQ-12 score did not compromise predictive accuracy when compared to the full KCCQ score. We conclude that the KCCQ or, given its lesser time to complete, the KCCQ-12 should be the preferred PROM in settings where PROMs are intended to extend inferences regarding future morbid and mortal outcomes in HF.

\section{Supplementary Material}

Refer to Web version on PubMed Central for supplementary material.

\section{Acknowledgments}

Funding: This research was supported by research funds from the National Institutes of Health (NIH; RC2HL102222). Drs. Larue and Vader were supported in part by NIH grant U10 HL110309. Dr. Yee was supported in part by the Mentors in Medicine Program, Division of Medical Education, Department of Internal Medicine, Washington University School of Medicine.

\section{References}

1. Garin O, Herdman M, Vilagut G, Ferrer M, Ribera A, Rajmil L, Valderas JM, Guillemin F, Revicki D, Alonso J. Assessing health-related quality of life in patients with heart failure: a systematic, standardized comparison of available measures. Heart Fail Rev 2014;19:359-367. [PubMed: 23681849]

2. Kelkar AA, Spertus J, Pang P, Pierson RF, Cody RJ, Pina IL, Hernandez A, Butler J. Utility of Patient-Reported Outcome Instruments in Heart Failure. JACC Heart Fail 2016;4:165-175. [PubMed: 26874386]

3. Green CP, Porter CB, Bresnahan DR, Spertus JA. Development and evaluation of the Kansas City Cardiomyopathy Questionnaire: a new health status measure for heart failure. J Am Coll Cardiol 2000;35:1245-1255. [PubMed: 10758967]

4. Rector TS, Cohn JN. Assessment of patient outcome with the Minnesota Living with Heart Failure questionnaire: reliability and validity during a randomized, double-blind, placebo-controlled trial of pimobendan. Pimobendan Multicenter Research Group. Am Heart J 1992;124:1017-1025.

[PubMed: 1529875]

5. Rector TS, Kubo SH, Cohn JN. Validity of the Minnesota Living with Heart Failure questionnaire as a measure of therapeutic response to enalapril or placebo. Am J Cardiol 1993;71:1106-1107.

[PubMed: 8475878]

6. Joseph SM, Novak E, Arnold SV, Jones PG, Khattak H, Platts AE, Davila-Roman VG, Mann DL, Spertus JA. Comparable performance of the Kansas City Cardiomyopathy Questionnaire in patients with heart failure with preserved and reduced ejection fraction. Circ Heart Fail 2013;6:1139-1146. [PubMed: 24130003]

7. Rector TS, Carson PE, Anand IS, McMurray JJ, Zile MR, McKelvie RS, Komajda M, Kuskowski M, Massie BM; I-PRESERVE Trial Investigators. Assessment of long-term effects of irbesartan on heart failure with preserved ejection fraction as measured by the minnesota living with heart failure questionnaire in the irbesartan in heart failure with preserved systolic function (I-PRESERVE) trial. Circ Heart Fail 2012;5:217-225. [PubMed: 22267751]

8. Myers J, Zaheer N, Quaglietti S, Madhavan R, Froelicher V, Heidenreich P. Association of functional and health status measures in heart failure. J Card Fail 2006;12:439-445. [PubMed: 16911910]

9. Soto GE, Jones P, Weintraub WS, Krumholz HM, Spertus JA. Prognostic value of health status in patients with heart failure after acute myocardial infarction. Circulation 2004;110:546-551. [PubMed: 15262843] 
10. Parissis JT, Nikolaou M, Farmakis D, Paraskevaidis IA, Bistola V, Venetsanou K, Katsaras D, Filippatos G, Kremastinos DT. Self-assessment of health status is associated with inflammatory activation and predicts long-term outcomes in chronic heart failure. Eur J Heart Fail 2009;11:163169. [PubMed: 19168514]

11. Spertus JA. Evolving applications for patient-centered health status measures. Circulation 2008;118:2103-2110. [PubMed: 19001034]

12. Zakeri R, Levine JA, Koepp GA, Borlaug BA, Chirinos JA, LeWinter M, VanBuren P, DavilaRoman VG, de Las Fuentes L, Khazanie P, Hernandez A, Anstrom K, Redfield MM. Nitrate's effect on activity tolerance in heart failure with preserved ejection fraction trial: rationale and design. Circ Heart Fail 2015;8:221-228. [PubMed: 25605640]

13. Napier R, McNulty SE, Eton DT, Redfield MM, AbouEzzeddine O, Dunlay SM. Comparing Measures to Assess Health-Related Quality of Life in Heart Failure With Preserved Ejection Fraction. JACC Heart Fail 2018;6:552-560. [PubMed: 29885952]

14. Spertus JA, Jones PG. Development and Validation of a Short Version of the Kansas City Cardiomyopathy Questionnaire. Circ Cardiovasc Qual Outcomes 2015;8:469-476. [PubMed: 26307129]

15. Guller U, DeLong ER. Interpreting statistics in medical literature: a vade mecum for surgeons. J Am Coll Surg 2004;198:441-458. [PubMed: 14992748]

16. Pencina MJ, D'Agostino RB Sr., D'Agostino RB Jr., Vasan RS. Evaluating the added predictive ability of a new marker: from area under the ROC curve to reclassification and beyond. Stat Med 2008;27:157-172; discussion 207-212. [PubMed: 17569110]

17. Pencina MJ, D'Agostino RB Sr., Steyerberg EW. Extensions of net reclassification improvement calculations to measure usefulness of new biomarkers. Stat Med 2011;30:11-21. [PubMed: 21204120]

18. Heidenreich PA, Albert NM, Allen LA, Bluemke DA, Butler J, Fonarow GC, Ikonomidis JS, Khavjou O, Konstam MA, Maddox TM, Nichol G, Pham M, Piña IL, Trogdon JG; American Heart Association Advocacy Coordinating Committee; Council on Arteriosclerosis, Thrombosis and Vascular Biology; Council on Cardiovascular Radiology and Intervention; Council on Clinical Cardiology; Council on Epidemiology and Prevention; Stroke Council. Circ Heart Fail 2013;6:606-619. [PubMed: 23616602]

19. Blecker S, Paul M, Taksler G, Ogedegbe G, Katz S. Heart failure-associated hospitalizations in the United States. J Am Coll Cardiol 2013;61:1259-1267. [PubMed: 23500328]

20. Spertus J, Peterson E, Conard MW, Heidenreich PA, Krumholz HM, Jones P, McCullough PA, Pina I, Tooley J, Weintraub WS, Rumsfeld JS; Cardiovascular Outcomes Research Consortium. Monitoring clinical changes in patients with heart failure: a comparison of methods. Am Heart J 2005;150:707-715. [PubMed: 16209970]

21. Spertus J. Barriers to the Use of Patient-Reported Outcomes in Clinical Care. Circ Cardiovasc Qual Outcomes 2014;7:2-4. [PubMed: 24425704]

22. Weldring T, Smith SM. Patient-Reported Outcomes (PROs) and Patient-Reported Outcome Measures (PROMs). Health Serv Insights 2013;6:61-68. [PubMed: 25114561]

23. Pokharel Y, Khariton Y, Tang Y, Nassif ME, Chan PS, Arnold SV, Jones PG, Spertus JA. Association of Serial Kansas City Cardiomyopathy Questionnaire Assessments With Death and Hospitalization in Patients With Heart Failure With Preserved and Reduced Ejection Fraction: A Secondary Analysis of 2 Randomized Clinical Trials. JAMA Cardiol 2017;2:1315-1321. [PubMed: 29094152] 


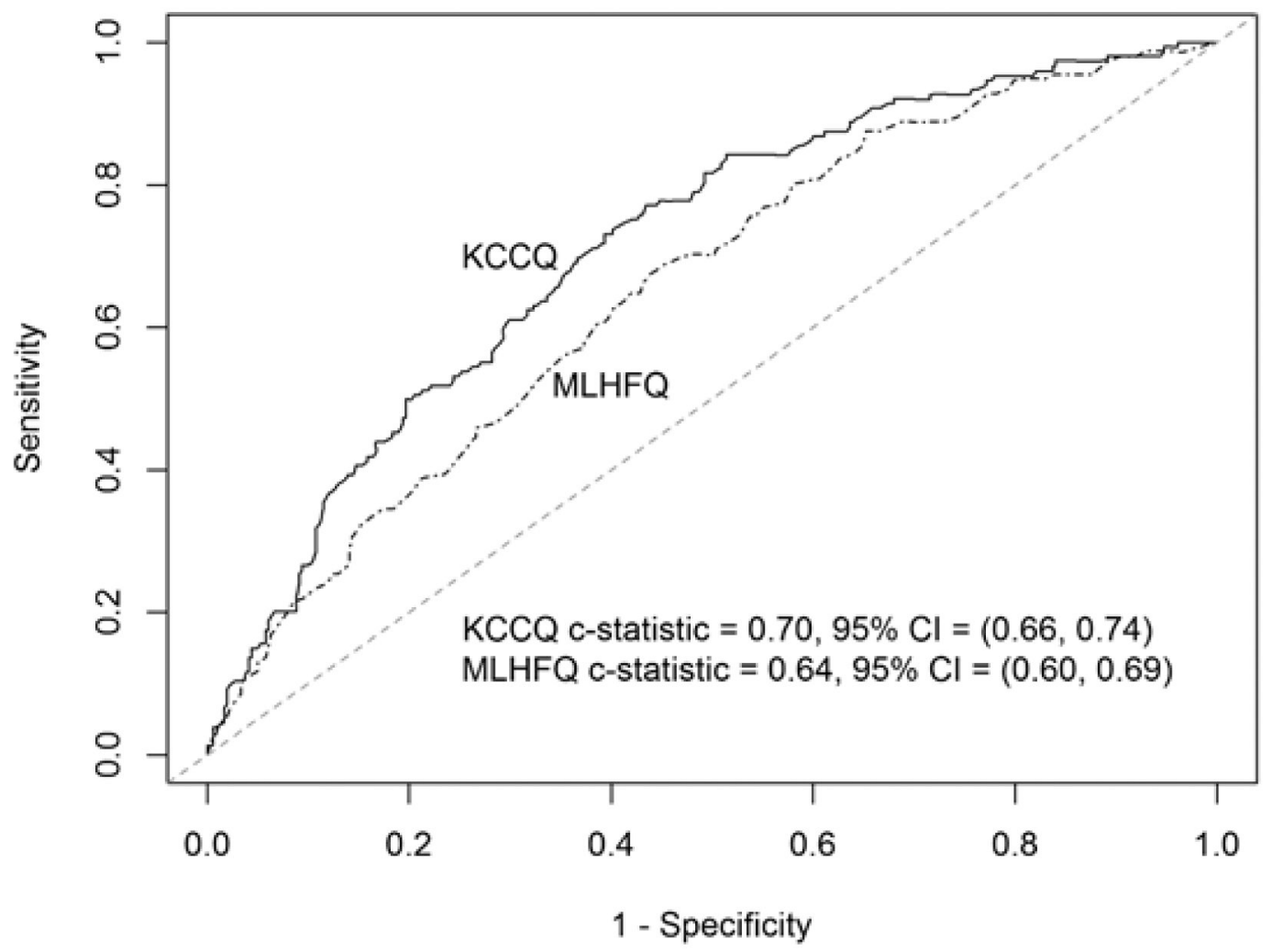

Figure 1.

Survival-based ROC curve for HFrEF.

$\mathrm{KCCQ}=$ Kansas City Cardiomyopathy Questionnaire; MLHFQ = Minnesota Living with Heart Failure Questionnaire; CI = Confidence interval. 


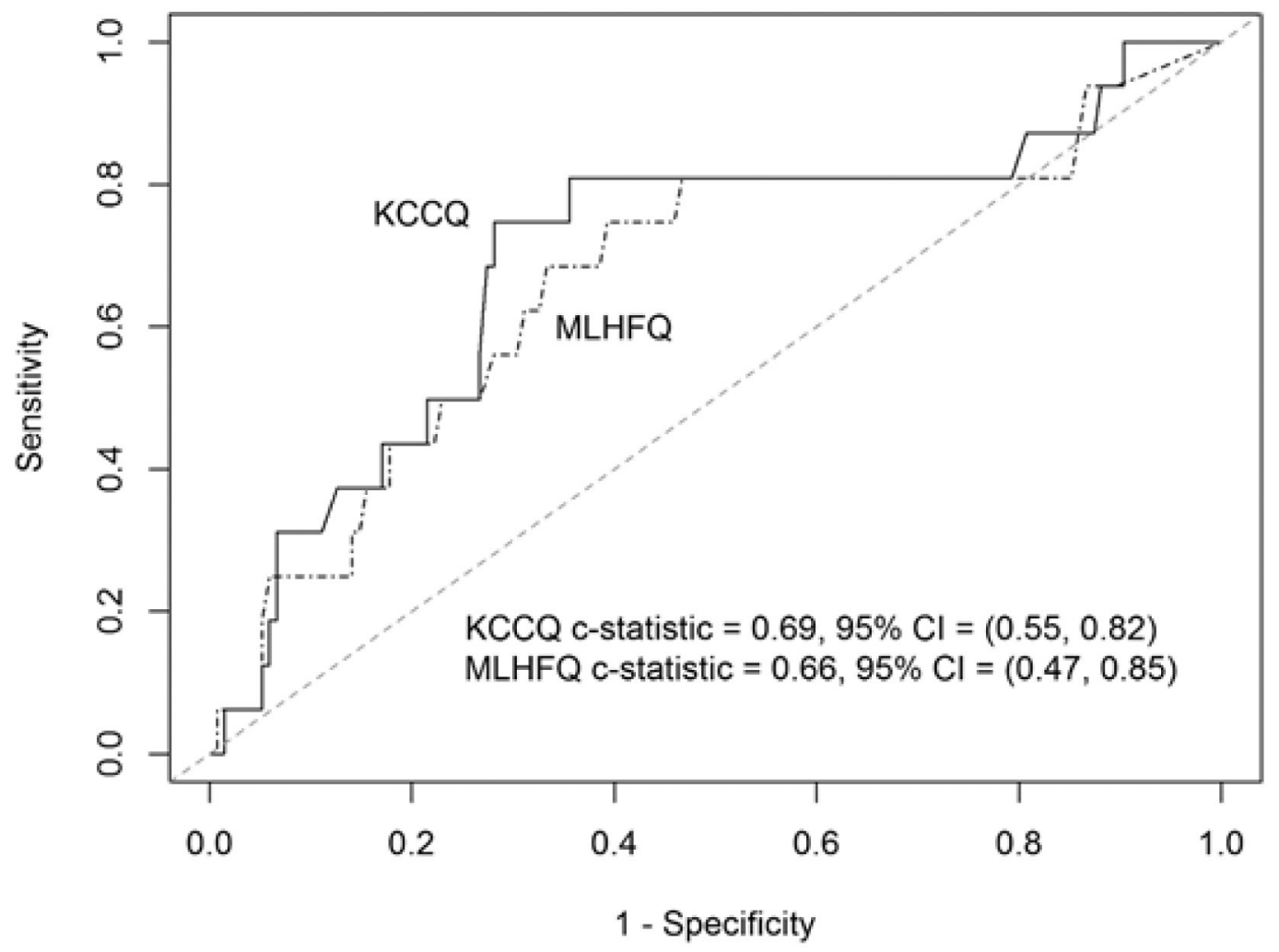

Figure 2.

Survival-based ROC curve for HFpEF

$\mathrm{KCCQ}=$ Kansas City Cardiomyopathy Questionnaire; MLHFQ = Minnesota Living with Heart Failure Questionnaire; CI = Confidence interval. 
Table 1.

Selected characteristics by KCCQ category

\begin{tabular}{|lcccccc|}
\hline \multicolumn{1}{|c}{ Variable } & $\begin{array}{c}\text { Overall } \\
(\mathbf{N = 7 3 8})\end{array}$ & $\begin{array}{c}\text { KCCQ 0-25 } \\
(\mathbf{N = 9 9})\end{array}$ & $\begin{array}{c}\text { KCCQ 26-50 } \\
\text { (N=216) }\end{array}$ & $\begin{array}{c}\text { KCCQ 51-75 } \\
\text { (N=198) }\end{array}$ & $\begin{array}{c}\text { KCCQ 76-100 } \\
\text { (N=225) }\end{array}$ & P-value \\
\hline Age At Enrollment (Years) & $54.78 \pm 13.08$ & $55.33 \pm 12.12$ & $53.97 \pm 11.95$ & $54.40 \pm 13.32$ & $55.65 \pm 14.28$ & 0.54 \\
Men & $494(67 \%)$ & $72(73 \%)$ & $151(70 \%)$ & $140(71 \%)$ & $131(58 \%)$ & 0.011 \\
White & $540(74 \%)$ & $61(62 \%)$ & $161(76 \%)$ & $151(77 \%)$ & $167(75 \%)$ & 0.05 \\
BMI (kg/m²) & $31.75 \pm 8.19$ & $32.42 \pm 8.83$ & $32.25 \pm 8.22$ & $31.92 \pm 7.86$ & $30.83 \pm 8.11$ & 0.22 \\
Heart rate (beats/min) & $77.49 \pm 15.12$ & $84.30 \pm 18.42$ & $79.21 \pm 14.23$ & $77.08 \pm 15.54$ & $73.20 \pm 12.45$ & $<.001$ \\
Systolic BP (mmHg) & $114.34 \pm 19.00$ & $111.54 \pm 22.04$ & $112.59 \pm 17.64$ & $113.96 \pm 18.18$ & $117.56 \pm 19.19$ & 0.014 \\
eGFR (mL/min/1.73m $\left.{ }^{2}\right)$ & $72.98 \pm 27.31$ & $66.19 \pm 26.65$ & $70.92 \pm 25.59$ & $73.37 \pm 29.81$ & $77.62 \pm 26.21$ & 0.003 \\
Sodium (mEq/L) & $138.98 \pm 3.69$ & $137.69 \pm 4.33$ & $138.74 \pm 3.84$ & $139.27 \pm 3.63$ & $139.55 \pm 3.10$ & $<.001$ \\
Prior MI & $262(36 \%)$ & $42(42 \%)$ & $86(40 \%)$ & $68(35 \%)$ & $66(29 \%)$ & 0.05 \\
Presence of AF & $243(33 \%)$ & $46(47 \%)$ & $91(43 \%)$ & $59(31 \%)$ & $47(21 \%)$ & $<.001$ \\
Prior coronary angioplasty & $189(26 \%)$ & $33(33 \%)$ & $64(30 \%)$ & $45(23 \%)$ & $47(21 \%)$ & 0.038 \\
Diabetes mellitus & $242(33 \%)$ & $47(47 \%)$ & $84(39 \%)$ & $58(29 \%)$ & $53(24 \%)$ & $<.001$ \\
Hypertension & $430(58 \%)$ & $71(72 \%)$ & $132(61 \%)$ & $113(57 \%)$ & $114(51 \%)$ & 0.003 \\
Hyperlipidemia & $334(45 \%)$ & $56(57 \%)$ & $100(46 \%)$ & $83(42 \%)$ & $95(42 \%)$ & 0.08 \\
Prior stroke/TIA & $85(12 \%)$ & $18(18 \%)$ & $31(14 \%)$ & $18(9 \%)$ & $18(8 \%)$ & 0.021 \\
Smoker & $462(64 \%)$ & $60(61 \%)$ & $151(71 \%)$ & $130(67 \%)$ & $121(55 \%)$ & 0.003 \\
\hline
\end{tabular}

KCCQ, Kansas City Cardiomyopathy Questionnaire; BMI, body mass index; BP, blood pressure; eGFR, estimated glomerular filtration rate; MI, myocardial infarction; $\mathrm{AF}$, atrial fibrillation; TIA, transient ischemic attack 
Table 2.

Selected characteristics by MLHFQ category

\begin{tabular}{|lcccccc|}
\hline \multicolumn{1}{|c}{ Variable } & $\begin{array}{c}\text { Overall } \\
(\mathbf{N = 7 3 8})\end{array}$ & $\begin{array}{c}\text { MLHFQ 0-26 } \\
(\mathbf{N = 2 1 1})\end{array}$ & $\begin{array}{c}\text { MLHFQ 27-52 } \\
(\mathbf{N = 1 6 7})\end{array}$ & $\begin{array}{c}\text { MLHFQ 53-78 } \\
(\mathbf{N = 2 1 5})\end{array}$ & $\begin{array}{c}\text { MLHFQ 79-105 } \\
(\mathbf{N = 1 4 5})\end{array}$ & P-value \\
\hline Age At Enrollment (Years) & $54.78 \pm 13.08$ & $56.26 \pm 14.31$ & $54.07 \pm 14.82$ & $55.54 \pm 11.03$ & $52.31 \pm 11.50$ & 0.028 \\
Men & $494(67 \%)$ & $125(59 \%)$ & $117(70 \%)$ & $156(73 \%)$ & $96(66 \%)$ & 0.025 \\
White & $540(74 \%)$ & $149(71 \%)$ & $133(80 \%)$ & $168(79 \%)$ & $90(63 \%)$ & 0.003 \\
BMI (kg/m²) & $31.75 \pm 8.19$ & $30.70 \pm 8.44$ & $31.85 \pm 7.62$ & $32.55 \pm 8.29$ & $31.98 \pm 8.22$ & 0.13 \\
Heart rate (beats/min) & $77.49 \pm 15.12$ & $73.67 \pm 14.53$ & $76.68 \pm 14.78$ & $78.97 \pm 14.95$ & $81.78 \pm 15.32$ & $<.001$ \\
Systolic BP (mmHg) & $114.34 \pm 19.00$ & $116.63 \pm 18.56$ & $116.33 \pm 19.13$ & $110.79 \pm 17.25$ & $113.92 \pm 21.18$ & 0.006 \\
eGFR (mL/min/1.73m²) & $72.98 \pm 27.31$ & $76.17 \pm 26.81$ & $74.67 \pm 29.90$ & $71.12 \pm 26.04$ & $69.15 \pm 26.32$ & 0.07 \\
Sodium (mEq/L) & $138.98 \pm 3.69$ & $139.44 \pm 3.17$ & $139.47 \pm 3.59$ & $139.04 \pm 3.68$ & $137.69 \pm 4.20$ & $<.001$ \\
Prior MI & $262(36 \%)$ & $65(31 \%)$ & $55(33 \%)$ & $85(40 \%)$ & $57(39 \%)$ & 0.19 \\
Presence of AF & $243(33 \%)$ & $45(21 \%)$ & $52(32 \%)$ & $91(43 \%)$ & $55(38 \%)$ & $<.001$ \\
Prior coronary angioplasty & $189(26 \%)$ & $41(19 \%)$ & $41(25 \%)$ & $61(29 \%)$ & $46(32 \%)$ & 0.037 \\
Diabetes mellitus & $242(33 \%)$ & $47(22 \%)$ & $52(31 \%)$ & $78(36 \%)$ & $65(45 \%)$ & $<.001$ \\
Hypertension & $430(58 \%)$ & $114(54 \%)$ & $98(59 \%)$ & $113(53 \%)$ & $105(72 \%)$ & $<.001$ \\
Hyperlipidemia & $334(45 \%)$ & $87(41 \%)$ & $71(43 \%)$ & $99(46 \%)$ & $77(53 \%)$ & 0.14 \\
Prior stroke/TIA & $85(12 \%)$ & $18(9 \%)$ & $18(11 \%)$ & $28(13 \%)$ & $21(14 \%)$ & 0.29 \\
Smoker & $462(64 \%)$ & $115(56 \%)$ & $108(65 \%)$ & $145(70 \%)$ & $94(65 \%)$ & 0.025 \\
\hline
\end{tabular}

MLHFQ, Minnesota Living with Heart Failure Questionnaire; BMI, body mass index; BP, blood pressure; eGFR, estimated glomerular filtration rate; MI, myocardial infarction; $\mathrm{AF}$, atrial fibrillation; TIA, transient ischemic attack 
Table 3.

Multivariable Cox proportional hazards model for risk of death/Transplant/VAD implant, KCCQ

\begin{tabular}{|llll|}
\hline Variable & HR & 95\% CI & p-value \\
\hline KCCQ (per 5 units) & 0.894 & $(0.864,0.924)$ & $<.001$ \\
\hline Age at enrollment (per 1 unit) & 1.005 & $(0.990,1.020)$ & 0.51 \\
\hline Women & 0.625 & $(0.421,0.927)$ & 0.019 \\
\hline White & 1.154 & $(0.788,1.690)$ & 0.46 \\
\hline BMI (per 1 unit) & 0.967 & $(0.944,0.991)$ & 0.007 \\
\hline Heart rate (per 1 unit) & 1.014 & $(1.004,1.024)$ & 0.005 \\
\hline Systolic BP (per 1 unit) & 0.986 & $(0.976,0.996)$ & 0.006 \\
\hline eGFR (per 1 unit) & 0.986 & $(0.979,0.992)$ & $<.001$ \\
\hline Sodium (per 1 unit) & 0.931 & $(0.897,0.965)$ & $<.001$ \\
\hline Prior MI & 0.922 & $(0.611,1.391)$ & 0.70 \\
\hline Presence of AF & 1.451 & $(1.037,2.032)$ & 0.030 \\
\hline Prior coronary angioplasty & 1.660 & $(1.083,2.544)$ & 0.020 \\
\hline Diabetes mellitus & 0.996 & $(0.708,1.400)$ & 0.98 \\
\hline Hypertension & 0.779 & $(0.540,1.124)$ & 0.18 \\
\hline Hyperlipidemia & 0.656 & $(0.461,0.935)$ & 0.020 \\
\hline Prior stroke/TIA & 2.015 & $(1.380,2.942)$ & $<.001$ \\
\hline Smoker & 0.769 & $(0.554,1.066)$ & 0.12 \\
\hline Ejection fraction (per 5 units) & 0.902 & $(0.845,0.963)$ & 0.002 \\
\hline & & & \\
\hline
\end{tabular}

KCCQ, Kansas City Cardiomyopathy Questionnaire; BMI, body mass index; BP, blood pressure; eGFR, estimated glomerular filtration rate; MI,

myocardial infarction; AF, atrial fibrillation; TIA, transient ischemic attack; VAD, ventricular assist device 
Table 4.

Multivariable Cox proportional hazards model for risk of death/Transplant/VAD implant, MLHFQ

\begin{tabular}{|llll|}
\hline Variable & HR & 95\% CI & p-value \\
\hline MLHFQ (per 5 units) & 1.077 & $(1.045,1.109)$ & $<.001$ \\
\hline Age at enrollment (per 1 unit) & 1.006 & $(0.992,1.021)$ & 0.41 \\
\hline Women & 0.642 & $(0.434,0.950)$ & 0.027 \\
\hline White & 1.078 & $(0.736,1.577)$ & 0.70 \\
\hline BMI (per 1 unit) & 0.972 & $(0.948,0.995)$ & 0.020 \\
\hline Heart rate (per 1 unit) & 1.018 & $(1.008,1.028)$ & $<.001$ \\
\hline Systolic BP (per 1 unit) & 0.984 & $(0.974,0.994)$ & 0.002 \\
\hline eGFR (per 1 unit) & 0.986 & $(0.979,0.992)$ & $<.001$ \\
\hline Sodium (per 1 unit) & 0.931 & $(0.898,0.965)$ & $<.001$ \\
\hline Prior MI & 0.994 & $(0.661,1.494)$ & 0.98 \\
\hline Presence of AF & 1.596 & $(1.145,2.226)$ & 0.006 \\
\hline Prior coronary angioplasty & 1.589 & $(1.040,2.428)$ & 0.032 \\
\hline Diabetes mellitus & 1.065 & $(0.759 .1 .497)$ & 0.71 \\
\hline Hypertension & 0.808 & $(0.561,1.165)$ & 0.25 \\
\hline Hyperlipidemia & 0.640 & $(0.449,0.913)$ & 0.014 \\
\hline Prior stroke/TIA & 2.046 & $(1.401,2.988)$ & $<.001$ \\
\hline Smoker & 0.754 & $(0.543,1.046)$ & 0.09 \\
\hline Ejection fraction (per 5 units) & 0.905 & $(0.847,0.966)$ & 0.003 \\
\hline
\end{tabular}

MLHFQ, Minnesota Living in Heart Failure Questionnaire; BMI, body mass index; BP, blood pressure; eGFR, estimated glomerular filtration rate; MI, myocardial infarction; AF, atrial fibrillation; TIA, transient ischemic attack; VAD, ventricular assist device 
Table 5.

Outcome frequency counts and Kaplan-Meier estimates, overall, HFrEF, HFpEF

\begin{tabular}{|c|c|c|c|}
\hline \multicolumn{4}{|c|}{ Overall } \\
\hline & Count $(\%)$ & KM 2-yr event rate & $95 \% \mathrm{CI}$ \\
\hline Combined Death/VAD/Transplant & $181(25 \%)$ & 0.241 & $(0.212,0.274)$ \\
\hline Death & $120(16 \%)$ & 0.161 & $(0.136,0.190)$ \\
\hline VAD & $62(8 \%)$ & 0.089 & $(0.070,0.112)$ \\
\hline Transplant & $36(5 \%)$ & 0.052 & $(0.038,0.072)$ \\
\hline Hospitalization & $415(56 \%)$ & 0.594 & $(0.557,0.631)$ \\
\hline \multicolumn{4}{|c|}{ HFrEF } \\
\hline & Count $(\%)$ & KM 2-yr event rate & $95 \% \mathrm{CI}$ \\
\hline Combined Death/VAD/Transplant & $156(30 \%)$ & 0.298 & $(0.261,0.340)$ \\
\hline Death & $101(20 \%)$ & 0.195 & $(0.163,0.233)$ \\
\hline VAD & $59(11 \%)$ & 0.122 & $(0.095,0.155)$ \\
\hline Transplant & $32(6 \%)$ & 0.068 & $(0.048,0.095)$ \\
\hline Hospitalization & $309(60 \%)$ & 0.646 & $(0.602,0.690)$ \\
\hline \multicolumn{4}{|c|}{ HFpEF } \\
\hline & Count $(\%)$ & KM 2-yr event rate & $95 \% \mathrm{CI}$ \\
\hline Combined Death/VAD/Transplant & $16(11 \%)$ & 0.106 & $(0.067,0.168)$ \\
\hline Death & $13(9 \%)$ & 0.087 & $(0.051,0.144)$ \\
\hline VAD & $1(1 \%)$ & 0.007 & $(0.001,0.049)$ \\
\hline Transplant & $2(1 \%)$ & 0.014 & $(0.003,0.054)$ \\
\hline Hospitalization & $72(48 \%)$ & 0.487 & $(0.409,0.570)$ \\
\hline
\end{tabular}

HFrEF, heart failure with reduced EF; HFpEF, heart failure with preserved EF; VAD, ventricular assist device; KM, Kaplan-Meier; CI, confidence interval 